\title{
PROL1 is essential for xenograft tumor development in mice injected with the human prostate cancer cell-line, LNCaP, and modulates cell migration and invasion
}

\author{
Amarnath Mukherjee ${ }^{1, \dagger}$, Augene Park ${ }^{1, \dagger}$, Kelvin Paul Davies ${ }^{1,2, *}$ \\ ${ }^{1}$ Department of Urology, Albert Einstein College of Medicine, Bronx, NY 10461, USA \\ ${ }^{2}$ Department of Physiology and Biophysics, Albert Einstein College of Medicine, Bronx, NY 10461, USA \\ *Correspondence: kelvin.davies@einsteinmed.org (Kelvin Paul Davies) \\ $\dagger$ These authors contributed equally. \\ Submitted: 28 July 2021 Accepted: 23 September 2021 Available online: 14 October 2021 Published: 10 February 2022
}

\begin{abstract}
Background and objective: A growing body of literature suggests modulated expression of members of the opiorphin family of genes (PROL1, SMR 3A and SMR 3B) is associated with cancer. Recently, overexpression of PROL1 was shown to be associated with prostate cancer, with evidence of a role in overcoming the hypoxic barrier that develops as tumors grow. The primary goal of the present studies was to support and expand evidence for a role of PROL1 in the development and progression of prostate cancer. Material and methods: We engineered knock-out of the opiorphin gene, PROL1, in LNCaP, an androgen-sensitive, human prostate cancer derived, cell-line. Using xenograft assays, we compared the ability of injected LNCaP PROL1 knock-out cell-lines to develop tumors in both castrated and intact male mice with the parental $\mathrm{LNCaP}$ and $\mathrm{LNCaP} P R O L 1$ overexpressing cell-lines. We used RNAseq to compare global gene expression between the parental and LNCaP PROL1 knock-out cell-lines. Wound closure and 3D spheroid invasion assays were used to compare cell motility and migration between parental LNCaP cells and LNCaP cells overexpressing of PROL1. Results: The present studies demonstrate that LNCaP cell-lines with consisitutive knock-out of PROL1 fail to develop tumors when injected into both castrated and intact male mice. Using RNAseq to compare global gene expression between the parental and LNCaP PROL1 knock-out cell-lines, we confirmed a role for PROL1 in regulating molecular pathways associated with angiogenesis and tumor blood supply, and also identified a potential role in pathways related to cell motility and migration. Through the use of wound closure and 3D spheroid invasion assays, we confirmed that overexpression of PROL1 in LNCaP cells leads to greater cell motility and migration compared to parental cells, suggesting that $P R O L 1$ overexpression results in a more invasive phenotype. Conclusion: Overall, our studies add to the growing body of evidence that opiorphin-encoding genes play a role in cancer development and progression. PROL1 is essential for establishment and growth of tumors in mice injected with $\mathrm{LNCaP}$ cells, and we provide evidence that PROL1 has a possible role in progression towards a more invasive, metastatic and castration resistant prostate cancer ( $\mathrm{PrCa})$.
\end{abstract}

Keywords: Cell motility; Cell invasiveness; Opiorphin; PROL1; Prostate cancer

\section{Introduction}

In the majority of countries throughout the world, prostate cancer $(\mathrm{PrCa})$ is the most common cancer affecting men; in 2018 there were approximately 1.3 million PrCa patients and 400,000 associated deaths [1]. Although the prognosis for patients with localized PrCa is good, metastatic, castration-resistant PrCa is invariably lethal [2]. Research aimed at increasing our understanding of the mechanisms and factors involved in the development of metastatic castration-resistant tumors has the potential to identify novel therapeutic strategies for PrCa.

In this regard, a growing body of evidence associates modulated expression of members of the opiorphin gene family (represented by PROL1, SMR3A and SMR3B) with several cancers [3-10], including a recently identified association between upregulated expression of $P R O L 1$ and $\mathrm{PrCa}$ [11]. The opiorphin genes encode peptides which act as potent endogenous neutral endopeptidase (NEP) inhibitors [12]. Opiorphin genes have previously been reported to act as master regulators of the hypoxic response in smooth muscle cells $[13,14]$, and PROL1 has recently been shown to regulate the expression of genes involved in the hypoxic response in PrCa cell-lines. Therefore, it has been suggested that PROL1 may play a role in overcoming the "hypoxic barrier", which results develops in the initial phase of tumor growth when uncontrolled cell proliferation often exceeds the ability to satisfy the oxygen demand from the preexisting blood vessel $[15,16]$. This usually occurs when the tumor exceeds a diameter of approximately $1 \mathrm{~mm}$ [17-19]. Overcoming the hypoxic barrier allows the tumor to develop, with increased invasion of local tissue and potential for metastasis to other parts of the body [20,21]. In addition, activation of the hypoxic response pathways has been associated with malignant progression towards castration resistant PrCa [22-24]. 
The primary goal of the present studies was to support and expand evidence for a role of PROL1 in the development and progression of PrCa. We generated a PROL1 knock-out LNCaP cell-line (LNCaP-ProL1-) and compared its ability to generate tumors when injected into mice with the parent (LNCaP) and PROL1 overexpressing (LNCaP-ProL1+) cell-lines. Global gene expression analysis confirmed a role for PROL1 in regulating molecular pathways associated with angiogenesis and tumor blood supply, but also identified a potential role in pathways related to cell motility and migration. We confirmed that overexpression of PROL1 in LNCaP-ProL1+ results in increased cell motility and migration, suggesting a role of PROL1 in both growth and progression of PrCa.

\section{Materials and methods}

2.1 Generation and maintenance of cell-lines (LNCaP, LNCaP-ProL1+, LNCaP-ProL1-)

LNCaP clone FGC (NCI-PBCF-CRL1740 (LNCaP Clone FGC)/ATCC ${ }^{\circledR}$ CRL-1740 ${ }^{T M}$; hereafter termed LNCaP) was obtained from the American Type Culture Collection (ATCC, Rockville, MD, USA). The generation and characterization of LNCaP cells overexpressing PROL1 (LNCaP-ProL1+) has been previously described [11]. PROL1 knockout cell-lines (LNCaP-ProL1) were generated using a commercially available PROL1 CRISPR knockout kit (Origene Technologies, Rockville, MD, USA); gRNA sequence GGACTTGGTGGAACCCATCT), according to manufacturers' protocol. Following antibiotic selection, it was confirmed that each colony was expressing GFP before pooling colonies and confirming knock-out of PROL1 across the non-clonal population. Control cell-lines for both LNCaP-ProL1+ and LNCaP-ProL1- were generated using a lentiviral control vector (Catalog \#: PS100093V, Origene Technologies) and a scramble CRISPR control vector (Catalog \#: GE100003, Origene Technologies), respectively. Cell-lines were maintained in Roswell Park Memorial Institute (RPMI) 1640 medium (Invitrogen, Carlsbad, CA, USA) with 10\% Fetal Bovine Serum (FBS, Thermo Fisher Scientific, Waltham, MA, USA), supplemented with $100 \mathrm{U} / \mathrm{mL}$ penicillin $\mathrm{G}$ and $100 \mathrm{ng} / \mathrm{mL}$ streptomycin (Invitrogen). In studies investigating the growth of cells in androgen depleted media the FBS was replaced with charcoal-stripped FBS (Sigma Aldrich, Burlington MA, USA).

All cell-lines were passaged on reaching $70 \%$ confluency (approximately 2 to 3 -day intervals) using a $0.25 \%$ Trypsin-EDTA solution (Thermo Fisher Scientific). Cell morphology and viability were monitored by microscopic observation using the CellTiter 96® AQueous One Solution Cell Proliferation Assay (MTS) from Promega (Madison, WI, USA) and regular Mycoplasma testing was performed (Universal Mycoplasma Detection Kit; ATCC). Growth rate was calculated as the average doubling time in cell number over a 24 -hour period when cells were in the loga- rithmic growth phase (performed in triplicate).

\subsection{Mouse xenograft studies}

Note: All animal studies were conducted in accordance with the Animal (Scientific Procedures) Act 1986 and approved by the Institutional Animal Care and Use Committee (IACUC) of Albert Einstein College of Medicine (protocol 20170801).

The mouse xenograft studies used the same methods and procedures as previously described [11]. Castrated and intact male nude mice were injected with $2.5 \times 10^{6}$ LNCaP-ProL1+, LNCaP-ProL1- and LNCaP cells and tumor size determined twice a week. The number of animals in each group are described in the Figure legends. Tumors were measured twice a week. Animals were euthanized 13weeks post-innoculation, or if they developed any tumors $>1000 \mathrm{~mm}^{3}$, through inhalation of $\mathrm{CO}_{2}$ to effect.

\subsection{Wound healing assay}

Wound healing assays were performed as previously described [25]. Briefly, cells were cultured in a $60 \mathrm{~mm}$ culture dish until reaching $90 \%-100 \%$ confluency at which point a scratch was created using a p200 pipette tip. Healing was monitored under an Olympus IX71 microscope equipped with Olympus DP72 camera. The distance between cell borders were determined using CellSens Standard imaging software (Olympus Life Science, Waltham, MA, USA).

\subsection{Spheroid tumor invasion assay}

The spheroid tumor invasion assay was adapted from a previously described method [26]. Briefly, 500-1000 cells were taken from culture and suspended in $20 \mu \mathrm{L}$ media, which was then pipetted onto the inner surface of 10 $\mathrm{cm}$ plate lid (40 drops in total). The lid was placed on a cell culture dish containing PBS and placed in an incubator. Spheroids were generated after approximately 3 days of culture and transferred into a microcentrifuge tube containing a mix of $100 \mu \mathrm{L}$ Matrigel (Corning Life Sciences, Teterboro, NJ, USA) and $100 \mu \mathrm{L} 3 \mathrm{mg} / \mathrm{mL}$ rat tail collagen I (Thermo Fisher Scientific). This mixture was embedded to the wells of a 24 well-plate (pre-treated with Matrigel) along with $1 \mathrm{~mL}$ cell culture media. After two weeks, invasion of the spheroids was determined through optical microscopy. Spheroids were determined to be invasive if a 2D layer of cells was observed growing around the spheroid.

\subsection{RNA Isolation, quantitative RT-PCR and RNAseq}

RNA was isolated from cell-lines using the RNeasy Plus Mini Kit (Qiagen, Hilden, Germany) and used to determine expression levels of PROL1 by quantitative-RT-PCR as previously described [11]. RNA was also sent to a commercial vendor (Novogene Corp., Sacramento, CA, USA) for performance of RNA-seq analysis of global gene expression as previously described. Similar criteria were used 
Table 1. Growth characteristics of the cell-lines used in these studies.

\begin{tabular}{lcc}
\hline Cell-line & $\begin{array}{c}\text { Growth rate }(10 \% \text { FBS }) \\
\text { Doubling time in days } \pm \text { Std. } \\
\text { error }\end{array}$ & $\begin{array}{c}\text { Growth rate (10\% } \\
\text { charcoal-stripped FBS) Doubling } \\
\text { time in days } \pm \text { Std. error }\end{array}$ \\
\hline LNCaP & $0.81 \pm 0.04$ & $3.01 \pm 0.06^{*}$ \\
LNCaP-ProL1+ & $0.71 \pm 0.08$ & $1.65 \pm 0.09^{*}$ \\
LNCap-ProL1- & $1.35 \pm 0.04$ & $13.2 \pm 0.04^{*}$ \\
\hline * a significant decrease in the growth rate of cell-line in charcoal-stripped compared to &
\end{tabular}

to identify differentially expressed genes (DEG) between LNCaP and LNCaP-ProL1- cell-lines as described in the analysis of DEG in LNCaP compared to LNCaP-ProL1+ cell-lines as previously described (i.e., cut-off criteria for differentially expressed genes was $>1$ Log2FoldChange or $<-1$ Log2FoldChange in gene expression with a $p$ value $<0.01[11])$. Gene ontology (GO) annotation analysis of DEG was performed using online analysis tools available from the database for annotation, visualization and integrated discovery (DAVID, vers 6.8, Homo sapien GOTERM_GO_Direct database $[27,28])$.

\subsection{Statistical analyses}

Statistical analyses were performed using either Microsoft Excel (Microsoft, Seattle, WA, USA) or Prism 8.2. (GraphPad Software, Inc., La Jolla, CA, USA). To determine statistical significance of two group comparisons, unpaired, two-tailed $t$-tests were performed, and results reported in Tables and Figures. Error bars represent standard deviation of mean (as described in figure legends).

\section{Results}

\subsection{Characterization of cell-lines}

Using qt-RT-PCR we confirmed knockout of PROL1 in our genetically engineered LNCaP cell-line (LNCaPProL1-). PROL1 was below the limit of detection in this assay. Prior studies have shown that in the LNCaP cellline engineered to overexpress LNCaP (LNCaP-ProL1+) there is $>28,560$-fold overexpression of $P R O L 1$ relative to LNCaP [11]. Under normal culture conditions (10\% FBS), although relative to $\mathrm{LNCaP}$ there was a trend for the doubling time to increase when PROL1 was overexpressed, and a trend for doubling time to increase with PROL1 knockout, this did not reach a significance of $p<0.005$ (Table 1, Supplementary Fig. 1). However, in media in which FBS was replaced by charcoal-stripped FBS, there was a significant decrease in the growth rate of all cell-lines (as has been previously observed for growth of androgen sensitive cell-lines [29]). As shown in Table 1, compared to LNCaP (where there was a 3.7-fold decrease in growth rate), the overexpression of PROL1 in LNCaP-ProL1+ partly mitigated this effect (a decrease in growth rate of 2.3-fold), whereas the PROL1 knockout in LNCaP-ProL1- exacerbated the effect (approximately a 9.8-fold decrease in growth rate, respectively).

\subsection{PROL1 expression is essential for tumor development} and growth in xenografted LNCaP cell-lines

Prior studies have shown that intact male mice injected with either LNCaP or LNCaP-ProL1+ cell-lines develop tumors $[11,30]$. In contrast, the LNCaP cell-line developed for the present studies, where PROL1 was knockedout (LNCaP-ProL1-), was unable to form tumors when injected into intact male mice $(N=5)$.

3.3 Global gene expression analysis confirms a role for PROL1 in regulating molecular pathways associated with angiogenesis and tumor blood supply, and identifies a potential role in cell migration

In order to identify molecular pathways regulated by PROL1 that may be involved in PrCa growth and progression, we compared differentially expressed genes (DEG) between the LNCaP-ProL1- and LNCaP cell-lines (as described under Materials and Methods, Section 2.5). We identified 1563 DEG, of which 891 had upregulated, and 672 downregulated expression in LNCaP-ProL1- versus LNCaP (Supplementary Table 1). In a previous study, using an identical method of analysis, 1110 DEG were identified when PROL1 was overexpressed in LNCaP cells [11]. As shown in Supplementary Table 2, 312 DEG were common between the two analyses (representing 20\% of the total DEG identified in the present study when PROL1 was knocked-out, and $28 \%$ of the DEG identified in the previous study when PROL1 was overexpressed cells [11]). As might be predicted, there was reciprocality in the fold-change in DEG when with PROL1 was overexpressed compared to knocked-out (i.e DEG that have increased levels of expression in LNCaP cells overexpressing PROL1 cells, have decreased expression in LNCaP cells with PROL1 knockout, and vice versa, as shown in Supplementary Table 2). For example, brain acid soluble protein 1 (BASP1, which is upregulated in several cancers and has been reported to promote tumor growth [31,32], is upregulated with $P R O L 1$ overexpression $(\log 2 \mathrm{FC}=6.32 ; p$ value, $1.4 \times 10^{-8}$ ) and downregulated with $P R O L 1$ knockout $\left(\log 2 \mathrm{FC}=-5.07 ; p\right.$-value, $\left.3.2 \times 10^{-60}\right)$. Similarly, semaphorin 4G (SEMA4G, which has been suggested as 
Table 2A. Gene ontology analysis of differentially expressed genes resulting from PROL1 knockout in LNCaP cells: angiogenesis/tumor blood supply/cell migration associated.

\begin{tabular}{|c|c|c|c|c|}
\hline Overrepresented ontological group & GO identifier & $\begin{array}{l}\text { \# Represented genes (1563. submitted, } \\
1305 \text { recognized) }\end{array}$ & Fold-enrichment & $p$-value \\
\hline \multicolumn{5}{|c|}{ Angiogenesis/ tumor blood supply (DAVID_BP_all) } \\
\hline Blood circulation & 0008015 & 45 & 1.54 & $4.23 \times 10^{-3}$ \\
\hline Regulation of blood circulation & 1903522 & 30 & 1.73 & $4.44 \times 10^{-3}$ \\
\hline Vasculature development & 0001944 & 52 & 1.47 & $4.83 \times 10^{-3}$ \\
\hline Circulatory system development & 0072359 & 74 & 1.35 & $6.07 \times 10^{-3}$ \\
\hline Blood vessel development & 0001568 & 49 & 1.47 & $6.41 \times 10^{-3}$ \\
\hline Blood vessel morphogenesis & 0048514 & 42 & 1.48 & $1.07 \times 10^{-2}$ \\
\hline Angiogenesis & 0001525 & 36 & 1.50 & $1.52 \times 10^{-2}$ \\
\hline Smooth muscle contraction & 0006939 & 11 & 4.80 & $2.20 \times 10^{-2}$ \\
\hline Regulation of blood pressure & 0008217 & 17 & 1.71 & $3.90 \times 10^{-2}$ \\
\hline Regulation of angiogenesis & 0045765 & 20 & 1.56 & $5.48 \times 10^{-2}$ \\
\hline \multicolumn{5}{|c|}{ Cell Migration Motility (DAVID_BP_all) } \\
\hline Cell motility & 0048870 & 112 & 1.46 & $3.85 \times 10^{-5}$ \\
\hline Cell migration & 0016477 & 98 & 1.44 & $2.35 \times 10^{-4}$ \\
\hline Regulation of cell motility & 2000145 & 65 & 1.53 & $6.28 \times 10^{-4}$ \\
\hline Positive regulation of cell migration & 0030335 & 45 & 1.71 & $1.36 \times 10^{-3}$ \\
\hline Positive regulation of cell motility & 2000147 & 40 & 1.69 & $1.38 \times 10^{-3}$ \\
\hline
\end{tabular}

tumor suppressor gene for colorectal cancer [33]) is downregulated with $P R O L 1$ overexpression $(\log 2 \mathrm{FC}=-4.8, p$ value, $9 \times 10^{-18}$ ) and is upregulated with $P R O L 1$ knockout $\left(\log 2 \mathrm{FC}=2.6, p\right.$-value, $\left.1.2 \times 10^{-13}\right)$.

The complete list of 1563 DEG associated with PROL1 knockdown, as well as the subset of 312 DEG identified as common between PROL1 knockdown and PROL overexpression, were submitted to DAVID for ontological analysis. The DAVID database recognized 1305 of the 1563 , and 271 of the $312 \mathrm{DEG}$, as unique and identifiable genes. Complete results of gene ontology analysis in biological functions and disease are shown in Supplementary Tables 3,4,5. As shown in Supplementary Table 3, there was significant overrepresentation of DEG in ontological groups related to cancer, including PrCa. Overall, PROL1 knockout resulted in modulated expression of 72 genes associated with cancer (13 of these genes were also represented in the subset of DEG common between PROL1 knockout and overexpression) (Supplementary Table 6).

In previous studies it has been demonstrated that overexpression of PROL1 in LNCaP cells regulates genes involved in aniogenesis and tumor blood supply [11]. This led to the hypothesis that PROL1 contributes to the development of PrCa by promoting the vascularization of developing tumors, overcoming the hypoxic barrier. This observation was confirmed in the present studies on LNCaP cell-lines with PROL1 knockdown, where there was significant overrepresentation of DEG in ontological groups related to aniogenesis and tumor blood supply (Table 2A and Supplementary Table 4). Even in the small subset of DEG in common between PROL1 knockout and PROL1 overexpression (312 DEG), these ontological groups were overrepresented, often with a greater level of statistical significance (Table 2B and Supplementary Table 5). These two separate investigations, generating mutually supportive evidence, provides a high level of confidence that PROL1 regulates pathways related to angiogenesis and blood supply to the tumor.

Our analysis also identified an unreported association between PROL1 expression and the regulation of genes involved in cell motility and migration (Table 2A,2B). Given that increased cell motility and migration are associated with PrCa invasion and metastasis, we conducted experiments to determine if PROL1 overexpression is associated with modulated motility and migration.

\subsection{Overexpression of PROL1 in LNCaP cells results in greater cell motility and migration}

The regulation of genes involved in cell motility and migration by $P R O L 1$ described above, led us to determine if $P R O L 1$ overexpression in $\mathrm{LNCaP}$ cells results in changes in phenotype indicative of greater motility and migration. Using a wound healing assay, we demonstrate that that LNCaP-ProL1+ cells have a 1.7-fold increase in the mean rate of wound healing compared to $\mathrm{LNCaP}$ (from approximately 103 to $172 \mathrm{~mm}$ per 24 hours, Fig. 1A). This increase in cell motility is comparable to results from wound healing assays that have been used to support a role for other genes in promoting invasiveness [34-36].

In addition, we performed a $3 \mathrm{D}$ spheroid invasion assay on both LNCaP and LNCaP-ProL1+. As shown in Fig. $1 \mathrm{~B}$, there was a significant increase in invasiveness rate 
Table 2B. Gene ontology analysis of differentially expressed genes in common between PROL1 knockout or PROL1 overexpression in LNCaP cells: angiogenesis/tumor blood supply/cell migration associated.

\begin{tabular}{lcccc}
\hline Overrepresented ontological group & GO identifier & $\begin{array}{c}\text { \# Represented genes (312 submitted, } \\
\text { 271 recognized) }\end{array}$ & Fold-enrichment & $p$-value \\
\hline Angiogenesis/tumor blood supply (DAVID_BP_all) & 0008015 & & & 2.46 \\
Blood circulation & 1903522 & 17 & 2.44 & $2.10 \times 10^{-2}$ \\
Regulation of blood circulation & 0001944 & 10 & 2.38 & $1.73 \times 10^{-3}$ \\
Vasculature development & 0072359 & 26 & 2.02 & $1.09 \times 10^{-3}$ \\
Circulatory system development & 0001568 & 19 & 2.42 & $9.09 \times 10^{-4}$ \\
Blood vessel development & 0048514 & 15 & 2.34 & $7.28 \times 10^{-3}$ \\
Blood vessel morphogenesis & 0001525 & 12 & 2.13 & $2.61 \times 10^{-2}$ \\
Angiogenesis & 0006939 & 5 & 4.31 & $2.74 \times 10^{-2}$ \\
Smooth muscle contraction & 0008217 & 7 & 3.00 & $2.94 \times 10^{-2}$ \\
Regulation of blood pressure & 0045765 & 7 & 2.31 & $8.26 \times 10^{-2}$ \\
Regulation of angiogenesis & & 27 & 1.67 & $1.04 \times 10^{-2}$ \\
Cell Migration Motility (DAVID_BP_all) & 0016477 & 29 & 1.59 & $1.35 \times 10^{-2}$ \\
Cell migration & 0048870 & & & \\
Cell motility & & 29 & & \\
\hline
\end{tabular}

in the LNCaP-ProL1+ compared to control LNCaP cells (2.6-fold, $p=0.01)$. This increase is comparable to results from other spheroid growth assays that have been used to support a role for other genes in promoting invasiveness [37-39].

\section{Discussion}

The studies presented here demonstrate that PROL1 expression is essential for development of xenografted tumors in mice injected with a castration sensitive, human PrCa cell-line (LNCaP). Our global gene expression analysis confirmed a previously reported role for PROL1 in regulating pathways in angiogenesis and blood supply [11]. In addition, $P R O L 1$ was also identified as a regulator of pathways involved in cell motility and migration. In vitro assays confirmed overexpression of PROL1 in LNCaP cells results in greater cell motility and migration. Overall, our studies add to the increasing body of evidence that modulated PROL1 expression is associated with PrCa and provides mechanistic insights in to its role in tumor development and progression.

Evidence for a role of opiorphin in cancer has been increasing ever since the publication in 2008 of a rank aggregation analysis to identify common genes which have modulated expression across different cancer types [4]. In the aggregated list of top-50 genes, 36 had been previously been implicated in cancer (often multiple cancers), with PROL1 a member of the other group of 14 genes, which were suggested as potential novel cancer genes deserving of further scrutiny. Based on the observation that cancer is often associated with modulated neutral endopeptidase (NEP) activity, and that the peptide products of opiorphin genes act as a potent NEP inhibitors, a review article in 2015 also suggested opiorphin may play a role in cancer development [40]. Since the publication of these reviews, several reports have described an association between opiorphin expression and cancer; including breast cancer [3,5], oropharyngeal squamous cell carcinoma $[6,7]$, head and neck cancer $[8,9]$, hepatocellular carcinoma[10] and more recently, $\mathrm{PrCa}$ [11]. In the abence of an effective antibody for direct detection of opiorphin protein expression, all of the reported studies, as well as those reported here, are limited to demonstrating an association between opiorphin gene expression and cancer.

The mouse xenograft model is widely used as an animal model of PCa development [41-45], and our studies demonstrate that PROL1 expression is essential for the development of tumors from injected LNCaP cells. Male mice injected with LNCaP and LNCaP-ProL1+ cells develop tumors, whereas mice injected with LNCaP-ProL1- cells do not. However, our in vitro studies demonstrate that under normal culture conditions, neither knockout or overexpression of PROL1 in LNCaP cells impacts growth rate. Therefore, failure of male mice injected with LNCaP-ProL1cells to develop tumors is not an effect of PROL1 on growth rate, but rather, the absence of an adaptive response allowing injected cells to establish and grow tumors.

To develop a hypothesis for the role played by PROL1 in tumor growth, we considered published research on the biological activity of opiorphins and the molecular pathways regulated by PROL1 described in the present study. Physiological studies have shown that opiorphin directly regulates vascular smooth muscle tone with dysregulated opiorphin expression associated with several pathophysiology's involving blood flow, such as hypertension [46], erectile dysfunction [47] and priapism [48]. At the molecular 

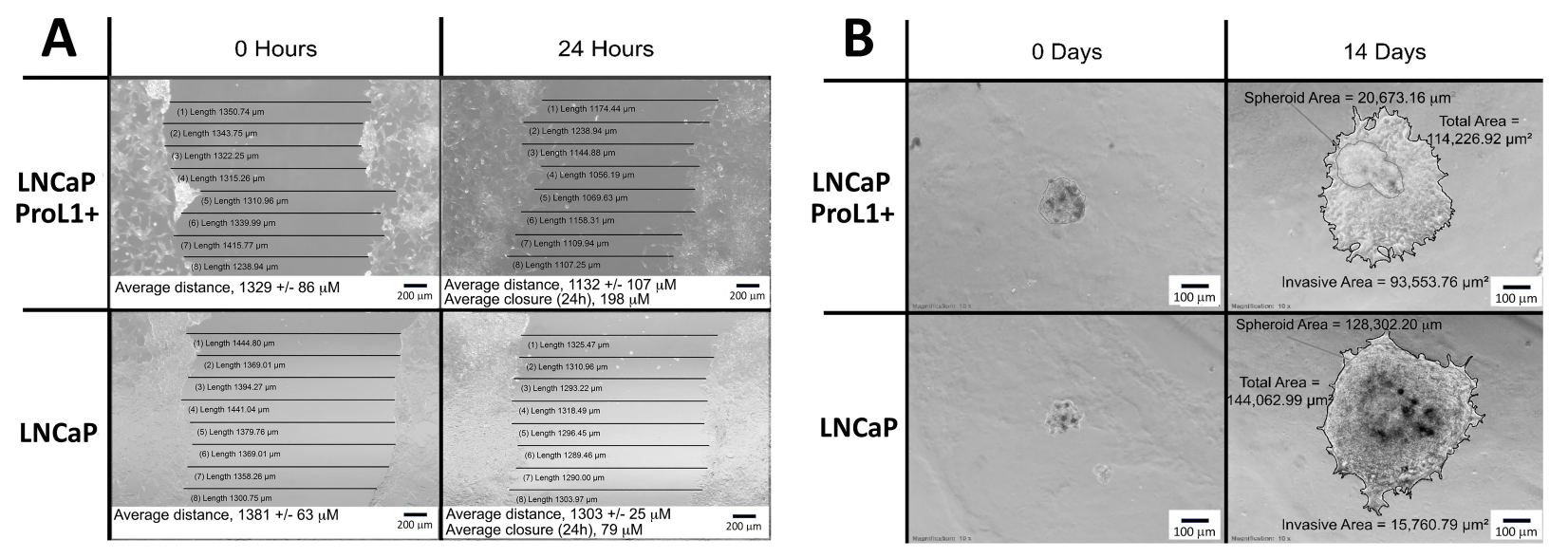

\begin{tabular}{|c|c|}
\hline Cell-line & $\begin{array}{c}\text { Wound closure } \\
\mu \mathrm{m} \text { after 24h (Std. Dev.) }\end{array}$ \\
\hline LNCaP-ProL1+ & $171^{\mathrm{A}} .6(72.5)^{*}$ \\
\hline LNCaP & $102.9(81.6)$ \\
\hline
\end{tabular}

\begin{tabular}{|c|c|c|c|}
\hline \multirow{2}{*}{ Cell-line } & \multicolumn{3}{|c|}{ Outcome } \\
\cline { 2 - 4 } & Invasive & $\begin{array}{c}\text { Non- } \\
\text { invasive }\end{array}$ & Total \\
\hline LNCaP-ProL1+ & 21 & 197 & 218 \\
\hline LNCaP & 8 & 198 & 206 \\
\hline Total & 29 & 395 & 424 \\
\hline
\end{tabular}

Fig. 1. Overexpression of $P R O L 1$ in LNCaP cells results in a more invasive phenotype. (A) A representative wound healing assay for LNCaP-ProL1+ and LNCaP cells is shown (upper panel). The width of the wound was measured at at least eight points per image. Data shown (lower panel) represents the mean rate of wound healing ( $\mu \mathrm{m}$ per 24 hours) \pm Std. Dev. of five independent experiments (with measurements performed in triplicate for each experiment). ${ }^{*}=p$-value $<0.001$. (B) A representative 3D spheroid invasion assay for LNCaP-ProL1+ and LNCaP cells is shown (upper panel). Spheroids were determined to be invasive if a 2D layer of cells was observed growing around the spheroid. Data shown (lower panel) represents the total number of invasive and non-invasive spheroids formed by LNCaP-ProL1+ and LNCaP after 2-weeks. LNCaP-ProL1+ cells generated significantly higher invasive according to the Fisher's exact test $(p<0.0001)$.

level, opiorphin has been shown to be a master regulator of hypoxic response pathways in smooth muscle cells $[13,14]$, and in the present studies we confirmed that in PrCa cells, PROL1 expression regulates pathways involved in angiogenesis and blood supply. Based on these observations, we hypothesize that $P R O L 1$ mediates an adaptive response to the hypoxic environment that develops as tumors grow, by activating pathways increasing tumor blood supply.

In addition, the present studies provide evidence that PROL1 may be also involved in the progression of localized PrCa towards a more invasive, metastatic and castration resistant cancer. Cell motility is a critical step in the progression to metastatic disease, and our global gene expression studies demonstrate that PROL1 regulates molecular pathways related to cell motility. Although there was no significant affect by PROL1 overexpression in $2 \mathrm{D}$ cell migration assays in Boyden chambers (data not shown), in wound closure and 3D spheroid growth assays, we confirmed that LNCaP-ProL1+ has significantly greater cell motility and migration than its parental cell-line. The difference to the $2 \mathrm{D}$ assay is potentially because the positive effects of PROL1 on cell motility and migration are only observed when cells face a environment more akin to an en- vironment present in a tumor, in vivo, and the $3 \mathrm{D}$ spheroid assay is generally considered a better tool to model the phenotypic and cellular heterogeneity, as well as microenvironmental aspects, of tumor growth in vivo [49].

Levels of PROL1 also appear to modulate the androgen-sensitivity of LNCaP cells. Although our in vitro studies demonstrate growth rate under normal culture conditions, when cells are grown in media in which FBS is replaced by charcoal stripped FBS (mimicking an androgenfree environment) LNCaP-ProL1+ cells show significant, 8-fold faster growth than LNCaP-ProL1- cells, suggesting overexpression of $P R O L 1$ results in reduced androgensensitivity. Further evidence that PROL1 is involved in the progression of $\mathrm{PrCa}$ cells from an androgen-sensitive to a castration resistant phenotype was provided through xenograft studies in castrated male mice. Remarkably, castrated mice injected with LNCaP-ProL + cells, in contrast to mice injected with LNCaP, developed tumors.

One of the goals of our research has been to document the role of PROL1 in regulating ontological groups of genes that may be involved in growth and development cancer (such as those involved in hypoxia, angiogenesis, cell motility and migration, etc). Although global gene ex- 
pression analysis by techniques such as RNA-seq may have a relatively poor correlation with the change of that specific gene at the protein level (about $40 \%$ of genes show both differential level of expression at the mRNA and protein level [50], when changes in ontologic groups of genes with differential expression at the mRNA level are compared with ontologic groups of proteins with differential expression, then the correlation is robust (mean $r=0.71$ ) [51]. Therefore, based on other studies, the correlation with ontological groups with differential protein expression would be predicted to be robust.

A limitation of our approach to identify the most significant ontological groups of genes regulated PROL1, is that we cannot rule out the involvement of individual genes involved in additional mechanisms of PrCa growth and development (in addition to hypoxia, angiogenesis, cell motility and migration). In future studies, where perhaps specific therapeutic targets are proposed based on data on changed expression level of a specific gene identified through global gene analysis, confirmation at the protein levels would be important. In addition, our studies have focused on the role of PROL1 in just one androgen-sensitive cell-line LNCaP. Although LNCaP is one of the most commonly utilized androgen-sensitive cell-lines used in in PrCa preclinical models [41], and are commonly the only cell-line in initial studies, in future studies the rigor of our findings would be improved if similar effects were found in additional PrCa cell-lines.

\section{Conclusions}

Overall, our studies add to the growing body of literature that opiorphin-encoding genes play a role in cancer development and progression. They are essential for establishment of PrCa tumors in mouse xenograft studies, and our evidence supports a possible role in progression towards a more invasive, metastatic and castration resistant PrCa. Targeting opiorphin expression or down-stream pathways regulated by opiorphins are potentially therapeutic strategies to prevent $\mathrm{PrCa}$ growth and progression.

\section{Abbreviations}

CSFBS, charcoal stripped fetal bovine serum; DAVID, database for annotation, visualization and integrated discovery; DEG, differentially expressed gene; FBS, fetal bovine serum; PrCa, prostate cancer; GO, gene ontology; GOC, gene ontology consortium; NEP, neutral endopeptidase; PROL1 (aka OPRPN), proline rich, lacrimal 1 (human opiorphin encoding gene); $h S M R 3 A / B$, human submaxillary gland androgen regulated protein 3 , homolog A (human opiorphin encoding gene).

\section{Author contributions}

Conceptualization-KPD; methodology-KPD, $\mathrm{AM}$ and $\mathrm{AP}$; formal analysis-KPD, AM and AP;
investigation-AM and $\mathrm{AP}$; resources-KPD; data curation-AP; writing - original draft preparation$\mathrm{KPD}$; writing - review and editing-KPD, AM and AP; visualization-KPD, $\mathrm{AM}$ and $\mathrm{AP}$; supervision-KPD; project administration-KPD; funding acquisition-KPD. All authors have read and agreed to the published version of the manuscript.

\section{Ethics approval and consent to participate}

All animal studies were conducted in accordance with the Animal (Scientific Procedures) Act 1986 and approved by the Institutional Animal Care and Use Committee (IACUC) of Albert Einstein College of Medicine (protocol 20170801).

\section{Acknowledgment}

Not applicable.

\section{Funding}

This research was funded by NIH/NIDDK, grant number DK107807 and DK109314 (PI: Kelvin P. Davies). The funders had no role in study design, data collection and analysis, decision to publish, or preparation of the manuscript.

\section{Conflict of interest}

The author declares no conflict of interest.

\section{Supplementary material}

Supplementary material associated with this article can be found, in the online version, at https://www.imrpre ss.com/journal/JOMH/18/2/10.31083/jomh.2021.131.

\section{References}

[1] Global Burden of Disease Cancer C, Fitzmaurice C, Abate D, Abbasi N, Abbastabar H, Abd-Allah F, et al. Global, Regional, and National Cancer Incidence, Mortality, Years of Life Lost, Years Lived With Disability, and Disability-Adjusted Life-Years for 29 Cancer Groups, 1990 to 2017: A Systematic Analysis for the Global Burden of Disease Study. Lancet. 2018; 392: 1789 1858.

[2] Wang G, Zhao D, Spring DJ, DePinho RA. Genetics and biology of prostate cancer. Genes \& Development. 2018; 32: 11051140.

[3] Lang Z, Wu Y, Pan X, Qu G, Zhang T. Study of differential gene expression between invasive multifocal/ multicentric and unifocal breast cancer. Journal of BUON. 2018; 23: 134-142.

[4] Pihur V, Datta S, Datta S. Finding common genes in multiple cancer types through meta-analysis of microarray experiments: a rank aggregation approach. Genomics. 2008; 92: 400-403.

[5] Lv X, He M, Zhao Y, Zhang L, Zhu W, Jiang L, et al. Identification of potential key genes and pathways predicting pathogenesis and prognosis for triple-negative breast cancer. Cancer Cell International. 2019; 19: 172

[6] Wu Q, Cao R, Chen J, Xie X. Screening and identification of biomarkers associated with clinicopathological parameters and prognosis in oral squamous cell carcinoma. Experimental and Therapeutic Medicine. 2019; 18: 3579-3587. 
[7] Koffler J, Holzinger D, Sanhueza GA, Flechtenmacher C, Zaoui $\mathrm{K}$, Lahrmann B, et al. Submaxillary gland androgen-regulated protein $3 \mathrm{a}$ expression is an unfavorable risk factor for the survival of oropharyngeal squamous cell carcinoma patients after surgery. European Archives of Oto-Rhino-Laryngology. 2013; 270: $1493-1500$.

[8] Grünow J, Rong C, Hischmann J, Zaoui K, Flechtenmacher C, Weber $\mathrm{K}$, et al. Regulation of submaxillary gland androgenregulated protein $3 \mathrm{a}$ via estrogen receptor 2 in radioresistant head and neck squamous cell carcinoma cells. Journal of Experimental \& Clinical Cancer Research. 2017; 36: 25.

[9] Thierauf J, Veit JA, Grunow J, Doscher J, Weissinger S, Whiteside T, et al. Expression of Submaxillary Gland Androgenregulated Protein 3A (SMR3A) in Adenoid Cystic Carcinoma of the Head and Neck. Anticancer Research. 2016; 36: 611-615.

[10] Wu J, Sun B, Ren N, Ye Q, Qin L. Genomic aberrations in hepatocellular carcinoma related to osteopontin expression detected by array-CGH. Journal of Cancer Research and Clinical Oncology. 2010; 136: 595-601.

[11] Mukherjee A, Park A, Wang L, Davies KP. Role of opiorphin genes in prostate cancer growth and progression. Future Oncology. 2021; 17: 2209-2223.

[12] Wisner A, Dufour E, Messaoudi M, Nejdi A, Marcel A, Ungeheuer M-, et al. Human Opiorphin, a natural antinociceptive modulator of opioid-dependent pathways. Proceedings of the National Academy of Sciences. 2006; 103: 17979-17984.

[13] Fu S, Tar MT, Melman A, Davies KP. Opiorphin is a master regulator of the hypoxic response in corporal smooth muscle cells. The FASEB Journal. 2014; 28: 3633-3644.

[14] Fu S, Davies KP. Opiorphin-dependent upregulation of CD73 (a key enzyme in the adenosine signaling pathway) in corporal smooth muscle cells exposed to hypoxic conditions and in corporal tissue in pre-priapic sickle cell mice. International Journal of Impotence Research. 2015; 27: 140-145.

[15] Siemann DW, Horsman MR. Modulation of the tumor vasculature and oxygenation to improve therapy. Pharmacology \& Therapeutics. 2015; 153: 107-124.

[16] Movsas B, Chapman JD, Horwitz EM, Pinover WH, Greenberg $\mathrm{RE}$, Hanlon $\mathrm{AL}$, et al. Hypoxic regions exist in human prostate carcinoma. Urology. 1999; 53: 11-18.

[17] Emami Nejad A, Najafgholian S, Rostami A, Sistani A, Shojaeifar S, Esparvarinha M, et al. The role of hypoxia in the tumor microenvironment and development of cancer stem cell: a novel approach to developing treatment. Cancer Cell International. 2021; 21: 62 .

[18] Folkman J. What is the Evidence that Tumors are Angiogenesis Dependent? Journal of the National Cancer Institute. 1990; 82: 4-7.

[19] Vaupel P, Kallinowski F, Okunieff P. Blood flow, oxygen and nutrient supply, and metabolic microenvironment of human tumors: a review. Cancer Research. 1989; 49: 6449-6465.

[20] Bacac M, Stamenkovic I. Metastatic Cancer Cell. Annual Review of Pathology: Mechanisms of Disease. 2008; 3: 221-247.

[21] Duffy M, McGowan P, Gallagher W. Cancer invasion and metastasis: changing views. The Journal of Pathology. 2008; 214: 283-293.

[22] Rudolfsson SH, Bergh A. Hypoxia drives prostate tumour progression and impairs the effectiveness of therapy, but can also promote cell death and serve as a therapeutic target. Expert Opinion on Therapeutic Targets. 2009; 13: 219-225.

[23] Vaupel P, Multhoff G. Fatal Alliance of Hypoxia-/HIF-1alphaDriven Microenvironmental Traits Promoting Cancer Progression. Advancesin Experimental Medicine and Biology. 2020; 1232: $169-176$

[24] O'Reilly D, Johnson P, Buchanan PJ. Hypoxia induced cancer stem cell enrichment promotes resistance to androgen depriva- tion therapy in prostate cancer. Steroids. 2019; 152: 108497.

[25] Martinotti S, Ranzato E. Scratch Wound Healing Assay. Methods in Molecular Biology. 2019; 26: 225-229.

[26] Berens EB, Holy JM, Riegel AT, Wellstein A. A Cancer Cell Spheroid Assay to Assess Invasion in a 3D Setting. Journal of Visualized Experiments. 2015; 53409.

[27] Huang DW, Sherman BT, Lempicki RA. Systematic and integrative analysis of large gene lists using DAVID bioinformatics resources. Nature Protocols. 2009; 4: 44-57.

[28] Huang DW, Sherman BT, Lempicki RA. Bioinformatics enrichment tools: paths toward the comprehensive functional analysis of large gene lists. Nucleic Acids Research. 2009; 37: 1-13.

[29] Lee SO, Dutt SS, Nadiminty N, Pinder E, Liao H, Gao AC. Development of an androgen-deprivation induced and androgen suppressed human prostate cancer cell line. The Prostate. 2007; 67: 1293-1300.

[30] Umekita Y, Hiipakka RA, Kokontis JM, Liao S. Human prostate tumor growth in athymic mice: inhibition by androgens and stimulation by finasteride. Proceedings of the National Academy of Sciences. 1996; 93: 11802-11807.

[31] Jaikumarr Ram A, Girija AS S, Jayaseelan VP, Arumugam P. Overexpression of BASP1 Indicates a Poor Prognosis in Head and Neck Squamous Cell Carcinoma. Asian Pacific Journal of Cancer Prevention. 2020; 21: 3435-3439.

[32] Tang H, Wang Y, Zhang B, Xiong S, Liu L, Chen W, et al. High brain acid soluble protein 1(BASP1) is a poor prognostic factor for cervical cancer and promotes tumor growth. Cancer Cell International. 2017; 17: 97.

[33] Wu S, Yuan W, Shen Y, Lu X, Li Y, Tian T, et al. The miR608 rs4919510 polymorphism may modify cancer susceptibility based on type. Tumor Biology. 2017; 39: 1010428317703819.

[34] Sohn HM, Kim B, Park M, Ko YJ, Moon YH, Sun JM, et al. Effect of CD133 overexpression on bone metastasis in prostate cancer cell line LNCaP. Oncology Letters. 2019; 18: 1189-1198.

[35] Sun C, Zhang G, Cheng S, Qian H, Li D, Liu M. URG11 promotes proliferation and induced apoptosis of $\mathrm{LNCaP}$ cells. International Journal of Molecular Medicine. 2019; 43: 2075-2085.

[36] Nakonechnaya AO, Shewchuk BM. Growth hormone enhances LNCaP prostate cancer cell motility. Endocrine Research. 2015; 40: 97-105.

[37] Thomas S, Chiriva-Internati M, Shah GV. Calcitonin receptorstimulated migration of prostate cancer cells is mediated by urokinase receptor-integrin signaling. Clinical \& Experimental Metastasis. 2007; 24: 363-377.

[38] Chin YR, Yuan X, Balk SP, Toker A. PTEN-Deficient Tumors Depend on AKT2 for Maintenance and Survival. Cancer Discovery. 2014; 4: 942-955.

[39] Li Y, Donmez N, Sahinalp C, Xie N, Wang Y, Xue H, et al. SRRM4 Drives Neuroendocrine Transdifferentiation of Prostate Adenocarcinoma under Androgen Receptor Pathway Inhibition. European Urology. 2017; 71: 68-78.

[40] Mizerska-Dudka M, Kandefer-Szerszeń M. Opioids, Neutral Endopeptidase, its Inhibitors and Cancer: is there a Relationship among them? Archivum Immunologiae Et Therapiae Experimentalis. 2015; 63: 197-205.

[41] Namekawa T, Ikeda K, Horie-Inoue K, Inoue S. Application of Prostate Cancer Models for Preclinical Study: Advantages and Limitations of Cell Lines, Patient-Derived Xenografts, and Three-Dimensional Culture of Patient-Derived Cells. Cells. 2019; 8: 74.

[42] Okada S, Vaeteewoottacharn K, Kariya R. Application of Highly Immunocompromised Mice for the Establishment of PatientDerived Xenograft (PDX) Models. Cells. 2019; 8: 889.

[43] Rea D, del Vecchio V, Palma G, Barbieri A, Falco M, Luciano A, et al. Mouse Models in Prostate Cancer Translational Research: from Xenograft to PDX. BioMed Research International. 2016; 
2016: 9750795.

[44] Dockhorn RJ, Green AW, Green E. Assessing the efficacy and safety of q. d. theophylline therapy: a multicenter study. Annals of Allergy. 1985; 55: 658-664.

[45] van Weerden WM, Romijn JC. Use of nude mouse xenograft models in prostate cancer research. The Prostate. 2000; 43: 263271.

[46] Calenda G, Tong Y, Kanika ND, Tar MT, Suadicani SO, Zhang $\mathrm{X}$, et al. Reversal of diabetic vasculopathy in a rat model of type 1 diabetes by opiorphin-related peptides. American Journal of Physiology-Heart and Circulatory Physiology. 2011; 301: H1353-H1359.

[47] Tong Y, Tar M, Monrose V, DiSanto M, Melman A, Davies KP. HSMR3a as a Marker for Patients with Erectile Dysfunction. Journal of Urology. 2007; 178: 338-343.

[48] Kanika ND, Tar M, Tong Y, Kuppam DSR, Melman A, Davies
KP. The mechanism of opiorphin-induced experimental priapism in rats involves activation of the polyamine synthetic pathway. American Journal of Physiology-Cell Physiology. 2009; 297: C916-C927.

[49] Thoma CR, Zimmermann M, Agarkova I, Kelm JM, Krek W. 3D cell culture systems modeling tumor growth determinants in cancer target discovery. Advanced Drug Delivery Reviews. 2014; 69-70: 29-41.

[50] Koussounadis A, Langdon SP, Um IH, Harrison DJ, Smith VA. Relationship between differentially expressed mRNA and mRNA-protein correlations in a xenograft model system. Scientific Reports. 2015; 5: 10775.

[51] Shankavaram UT, Reinhold WC, Nishizuka S, Major S, Morita $\mathrm{D}$, Chary KK, et al. Transcript and protein expression profiles of the NCI-60 cancer cell panel: an integromic microarray study. Molecular Cancer Therapeutics. 2007; 6: 820-832. 